\title{
The role of superficial wall stress and mechanical factors in scaffold failure: Protocol of the RANSOMED study
}

\author{
Juan Luis Gutiérrez-Chico, ${ }^{1,} \odot$, Lili $\operatorname{Liu}^{1} \oplus$, Miao $\mathrm{Chu}^{3}$, Ruiyan Zhang ${ }^{1}$, \\ Milosz J. Jaguszewski ${ }^{4} \oplus$, Giulio Makmur ${ }^{5}$, Tommaso Gori ${ }^{5}$, Shengxian $\mathrm{Tu}^{3}$ \\ ${ }^{1}$ Department of Cardiovascular Medicine, Ruijin Hospital, \\ Shanghai Jiao Tong University School of Medicine, Shanghai, China \\ ${ }^{2}$ CardioCare Heart Center, Marbella, Spain \\ ${ }^{3}$ Biomedical Instrument Institute, School of Biomedical Engineering, \\ Shanghai Jiao Tong University, Shanghai, China \\ ${ }^{4}$ First Cardiology Department, Gdansk University Hospital, Gdansk, Poland \\ ${ }^{5}$ University Medical Center, Mainz, Germany
}

This paper was guest edited by Prof. Robert J. Gil

\section{Background}

Bioresorbable scaffolds (BRS) were once heralded as the fourth revolution in interventional cardiology [1] (after percutaneous coronary intervention [PCI], bare-metal stents [BMS], and drugeluting stents [DES]). They were theoretically supposed to prevent restenosis as efficiently as DES, whilst dissipating the threat of late and very late stent thrombosis (VLST), because all intracoronary foreign bodies and potentially pro-inflammatory material completely disappeared from the coronary artery. Nonetheless, after initially promising results in pilot studies [2-6], BRS started to be questioned, as several observational studies and clinical trials consistently reported higher rates of scaffold thrombosis and device-oriented composite endpoint (cardiac death, target vessel myocardial infarction, and target vessel revascularization) than those observed in second generation DES [7-10]. Moreover, approximately two-thirds of the cases of scaffold thrombosis occurred at very late stages $[9,10]$, thus blasting the appealing hypothesis of transient scaffolding, whose risk of thrombosis vanishes in parallel with the bioresorption of the foreign body. Not even vasomotion was restored as initially expected [9], notwithstanding the encouraging results in the pilot studies $[2,11]$.

The reasons for the poor clinical performance of BRS are not fully elucidated, but they are most likely multifactorial. Procedural factors at the time of implantation play an important role, as suggested in the Coronary Slow-flow and Microvascular Disease Registry (MICAT), wherein the incidence of scaffold thrombosis was significantly reduced from $3.3 \%$ to $1.0 \%$ at 12 months after implementation of an angiographically guided BVS-dedicated implantation protocol [12]. A small series of 54 patients has reported $0 \%$ scaffold thrombosis at 12 months after an optical coherence tomography-guided implantation protocol, with tailored plaque preparation depending on the tissue characterization of the stenosis, and targeted optimization if needed [13]. Nonetheless the procedural factors do not fully explain the higher incidence of scaffold thrombosis: an excessively broad and non-selective indication to implant the

Address for correspondence: Prof. Juan Luis Gutiérrez-Chico, MD, PhD, FESC, FACC, Head of Interventional Cardiology, Ruijin Hospital, Shanghai Jiao Tong University School of Medicine, 197, $2^{\text {nd }}$ Ruijin Road, 200025 - Shanghai, China, tel: +34 615 319370, +86 13501974021, e-mail: juanluis.gutierrezchico@ictra.es

Received: 7.08.2021 Accepted: 7.11.2021 Early publication date: 11.01.2022

This article is available in open access under Creative Common Attribution-Non-Commercial-No Derivatives 4.0 International (CC BY-NC-ND 4.0) license, allowing to download articles and share them with others as long as they credit the authors and the publisher, but without permission to change them in any way or use them commercially. 


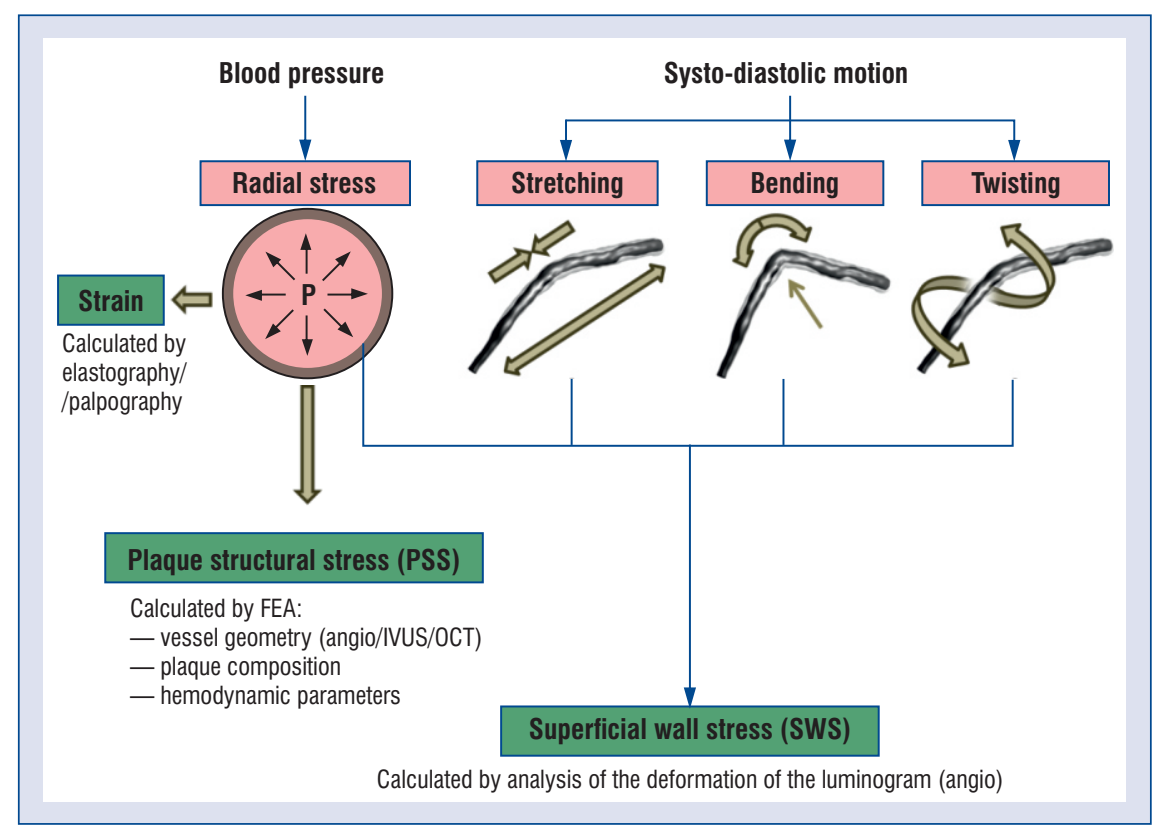

Figure 1. The four vectors of dynamic superficial wall stress and their relationship with other mechanical forces; FEA — finite element analysis; IVUS — intravascular ultrasound; OCT — optical coherence tomography.

device, disregarding the plaque characteristics, the eagerness of the operators to pioneer off-label indications, or an overly prolonged resorption process [14], protracted beyond the time slots defined in preclinical studies [15], might have played a relevant role in the failure of this promising technology.

Another intriguing phenomenon, named "dismantling", has also been described in some cases of very late scaffold thrombosis. It is defined as the total loss of structural integrity of the scaffold, with the presence of overhanging and floating struts [14], and it could partly explain very late clinical events, because a persistent scaffold with irregular loss of structural integrity resembles a model of stent fracture, which is a known risk factor for device failure [16-18]. The analysis of this phenomenon has inspired the socalled mechanical hypothesis of scaffold failure: the lack of structural integrity (dismantling) will occur at some point with any bioresorbable device, thus becoming a model of stent/scaffold fracture, which under specific mechanical stress conditions might result in increased inflammation, gross structural disarray of both the polymer and the vascular elements, and ultimately very late scaffold thrombosis or other clinical events.

A novel method to calculate dynamic superficial wall stress (SWS) has been developed recently, based on the dynamic lumen changes assessed in three-dimensional angiography [19-21]. Briefly, SWS summarizes the mechanical forces acting on the vessel wall in four different vectors: radial stress, stretching-compression, bending, and twisting (Fig. 1) [21]. It can be calculated by analysis of the deformation of the luminogram in conventional angiography [19-21]. We hypothesize that BRS implanted in coronary segments with low dynamic SWS will perform clinically better than BRS implanted in segments with high dynamic SWS, in which the repetitive stress can result in material fatigue from the moment of implantation and in mechanical instability once dismantling happens in the resorption process. We also hypothesize that the role played by the vectors depending on the systo-diastolic motion (i.e., stretching, bending, and twisting) on this process is likely to be more relevant than the vector depending on blood pressure (i.e., radial stress). These hypotheses have not been tested to date.

\section{Methods}

The aim of the RANSOMED (Resorption Aptness under Nocuous Stress: Outcomes and Mechanics for the Election of Device) study is to test the association between SWS in a coronary segment treated with BRS and very late clinical outcomes: target lesion failure, device failure, scaffold restenosis, and scaffold thrombosis. 


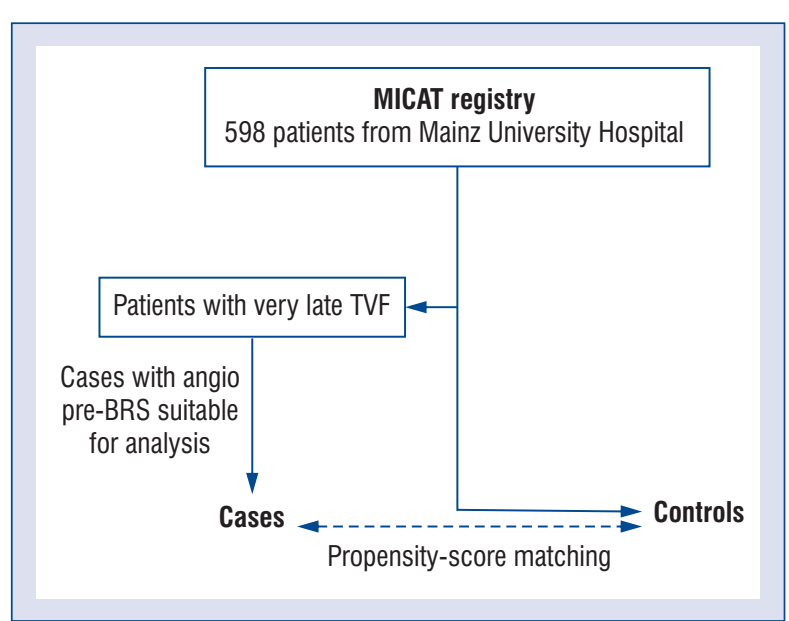

Figure 2. Study flow-chart; BRS- bioresorbable scaffold; TVF — target vessel failure.

\section{Study design and study population}

Post-hoc analysis of patients from Mainz enrolled in the MICAT study (NCT02180178) [12], following a case-control design. Patients with very late target vessel failure (TVF) will comprise the cases, while the controls will be selected from event-free BRS patients after propensity score matching (Fig. 2).

The inclusion criteria will be as follows: 1) Patients with one or more BRS implanted and enrolled in the MICAT registry [12]; 2) A TVF event between 12 months and 5 years after BRS implantation (cases) or event-free follow-up for 5 years (controls). TVF will be defined as cardiac death, vessel-related myocardial infarction, and target lesion revascularization. The exclusion criteria for both cases and controls will be as follows: 1) Angiography pre-implantation missing; 2) Documented scaffold rupture at the end of the implantation; 3) Hybrid BRS-DES revascularization in the same coronary segment; 4) Coronary angiography acquisition at $<12.5$ frames/s; 5) Coronary angiography deemed of insufficient quality due to vessel overlap, extreme foreshortening, or insufficient opacification of the target artery.

\section{Data management}

Anonymized clinical data and angiographies of the patients will be transferred to the online servers of the cardiovascular imaging core lab of Shanghai Jiao Tong University School of Medicine (clinical data) and of Med-X Research Institute (angiographies), in a secured and firewall-protected environment. Investigators responsible for the biomechanical analysis of the angiographies will be blinded to the clinical results and vice versa. The whole process of data handling will warrant patients' data confidentiality. The transferred data will not be used for any purpose other than the one explicitly described in this protocol without formally asking for permission from the MICAT investigators.

\section{Dynamic SWS calculation}

Angiography images in DICOM format will be analyzed at the central imaging core lab for conventional quantitative coronary angiography (QCA) analysis and dynamic SWS calculation. SWS at baseline (main target) and after BRS implantation (whenever available, secondary target) will be calculated at the scaffolded segment as previously described $[19,20]$. The following parameters will be collected to explore their association with clinical events:

- maximal SWS value in the scaffolded segment before PCI (pre-SWSmax);

- maximal SWS value in the scaffolded segment after BRS implantation (post-SWSmax);

- pre-post difference in maximal SWS $(\Delta \mathrm{SWS}$ $\max$;

- mean SWS value in the scaffolded segment before PCI (pre-SWSmean);

- mean SWS value in the scaffolded segment after BRS implantation (post-SWSmean);

- pre-post difference in mean SWS ( $\triangle \mathrm{SWSmean})$.

\section{Analysis of the four different vectors of SWS}

The role of the different components of SWS (radial stress, stretching, bending, and twisting) on the occurrence of very late TVF will be explored, taking the value of each vector provided by the software.

All the SWS vectors except twisting can be indirectly estimated using advanced software for conventional QCA analysis, which requires much lower quality and frame rate during the angiography acquisition than SWS for an accurate assessment. Advanced QCA software (AngioPlus Galley, Pulse Medical, Shanghai, China) can estimate plaque strain as a surrogate for radial stress, and the maximal differences in vessel angulation and segment length between systole and diastole as surrogates for bending and stretching, respectively. Plaque strain depends on blood pressure, whilst bending and stretching depend on systodiastolic motion; therefore, the different weight of these forces on the process of scaffold structural discontinuity, dismantling, and failure will be indirectly verified. 


\section{Study objectives}

The comparison of pre-SWSmax between patients with vs. without very late TVF will be the primary objective of the study.

Secondary objectives of the study will be as follows: a comparison of $\Delta$ SWSmax, parameters related to the mean dynamic SWS and each of the SWS vectors between patients with vs. without very late TVF, between patients with vs. without definite/probable scaffold thrombosis, and between patients with vs. without TVF at any time. The comparisons of SWS vectors will also include the QCA-derived plaque strain, bending, and stretching, as previously described.

The following exploratory objectives are defined: sensitivity, specificity, + predictive value, - predictive value, diagnostic accuracy, and area under the curve of the receiver-operator characteristics curve of the optimal cut-off value for the primary and secondary objectives, in the case of significant association.

Prespecified subgroup analysis will be performed for the primary and secondary objectives in which a significant association is found: angio- vs. optical coherence tomography-guided scaffold implantation, age groups, and main coronary territory (left anterior descending, left circumflex, and right coronary artery).

\section{Sample size calculation}

There are no preliminary data on this topic that enable an accurate sample size for the current study. We believe that the sample size of the MICAT study ( $\mathrm{n}=1305$ patients) and the reported event rate should provide a sufficient sample size to test the hypothesis of this study, which is essentially exploratory of the concept. In the unlikely case that the data from MICAT are not sufficient to provide statistical significance, but a clear trend can be derived from the analysis of the data, the investigators could consider increasing the study sample by the addition of other international BRS registries.

\section{Ethical aspects}

The study will be conducted in accordance with the ethical principles of the Declaration of Helsinki and consistent with ICH Good Clinical Practice as well as regulatory requirements. All data management will include anonymity of the participating patients and confidentiality of their clinical data. Access to the central database will be password-protected, and only the authorized investigators will be granted access. The angiogra- phy images in DICOM format will be anonymized and sent to the imaging core lab. The analysts of the angiography recordings will be unaware of the identity of the patients included in the study.

\section{Discussion}

The mechanical hypothesis explaining cases of very late scaffold failure after a model of stent/scaffold fracture occurring as a natural consequence of the resorption process in BRS has never been clinically tested to date. There are several reasons for this gap in the evidence. First of all, a prospective study with this aim would entail serious logistic challenges due to the low incidence of clinical events and the complex time-consuming evaluation of mechanical forces in the coronary artery. Such a study design would entail evaluation and thousands of angiographies through an expensive, cumbersome, and ponderous process to obtain a significant result. Therefore, the investigators have opted for an observational retrospective study to overcome these currently insurmountable logistic limitations. The study proposed herein follows a classical casecontrol design, which enables the investigators to focus on the few cases of scaffold failure (cases) and to compare them with matched controls. In this way the mechanical analysis is limited to a few cases (a maximum of 70), which is an affordable sample size and sufficient for a pilot exploratory study. However, this retrospective design entails a potentially severe limitation: the calculation of SWS demands very high quality angiography acquisition and ideally a high frame rate ( $>25$ frames/s). Neither this unusually high frame rate nor the high-quality requirements can be warranted for most cases in a retrospective study, so the accuracy of the estimation is uncertain, although it might be sufficient to show a meaningful signal. This is also the main reason to have included QCA-derived parameters that can be considered estimators of two of the vectors encompassed in the calculation of SWS: plaque strain - as an estimator of radial stress, and change in systo-diastolic angulation as an estimator of bending. These two parameters represent vectors that depend on blood pressure and on systo-diastolic motion, respectively, which we hypothesize to play the least and most relevant roles for the occurrence of material fatigue and scaffold failure in BRS from a mechanical point of view, respectively. These QCA-derived parameters are less quality-demanding and therefore might be instrumental to evaluate the mechanical hypothesis if the SWS analysis were unreliable. 
Conflict of interest: Juan Luis Gutiérrez-Chico received consultancy fees and speaker honoraria from Abbott, Asahi Intecc, and Boston Scientific. All other authors have nothing to disclose.

\section{References}

1. Wykrzykowska JJ, Onuma Y, Serruys PW. Vascular restoration therapy: the fourth revolution in interventional cardiology and the ultimate „rosy” prophecy. EuroIntervention. 2009; 5 (Suppl F): F7-F8, doi: 10.4244/EIJV5IFA1, indexed in Pubmed: 22100680.

2. Serruys PW, Ormiston JA, Onuma Y, et al. A bioabsorbable everolimus-eluting coronary stent system (ABSORB): 2-year outcomes and results from multiple imaging methods. Lancet. 2009; 373(9667): 897-910, doi: 10.1016/S0140-6736(09)60325-1, indexed in Pubmed: 19286089.

3. Ormiston JA, Serruys PW, Regar E, et al. A bioabsorbable everolimus-eluting coronary stent system for patients with single de-novo coronary artery lesions (ABSORB): a prospective open-label trial. Lancet. 2008; 371(9616): 899-907, doi: 10.1016/ S0140-6736(08)60415-8, indexed in Pubmed: 18342684.

4. Onuma Y, Serruys PW, Ormiston JA, et al. Three-year results of clinical follow-up after a bioresorbable everolimus-eluting scaffold in patients with de novo coronary artery disease: the ABSORB trial. EuroIntervention. 2010; 6(4): 447-453, doi: 10.4244/EIJ30V6I4A76, indexed in Pubmed: 20884431.

5. Dudek D, Onuma Y, Ormiston JA, et al. Four-year clinical follow-up of the ABSORB everolimus-eluting bioresorbable vascular scaffold in patients with de novo coronary artery disease: the ABSORB trial. EuroIntervention. 2012; 7(9): 1060-1061, doi: 10.4244/EIJV7I9A168, indexed in Pubmed: 21959320.

6. Onuma Y, Dudek D, Thuesen L, et al. Five-year clinical and functional multislice computed tomography angiographic results after coronary implantation of the fully resorbable polymeric everolimus-eluting scaffold in patients with de novo coronary artery disease: the ABSORB cohort A trial. JACC Cardiovasc Interv. 2013; 6(10): 999-1009, doi: 10.1016/j.jcin.2013.05.017, indexed in Pubmed: 24156961.

7. Capodanno D, Gori T, Nef H, et al. Percutaneous coronary intervention with everolimus-eluting bioresorbable vascular scaffolds in routine clinical practice: early and midterm outcomes from the European multicentre GHOST-EU registry. EuroIntervention. 2015; 10(10): 1144-1153, doi: 10.4244/EIJY14M07_11, indexed in Pubmed: 25042421.

8. Serruys PW, Chevalier B, Dudek D, et al. A bioresorbable everolimus-eluting scaffold versus a metallic everolimus-eluting stent for ischaemic heart disease caused by de-novo native coronary artery lesions (ABSORB II): an interim 1-year analysis of clinical and procedural secondary outcomes from a randomised controlled trial. Lancet. 2015; 385(9962): 43-54, doi: 10.1016/ S0140-6736(14)61455-0, indexed in Pubmed: 25230593.

9. Serruys PW, Chevalier B, Sotomi Y, et al. Comparison of an everolimus-eluting bioresorbable scaffold with an everolimus-eluting metallic stent for the treatment of coronary artery stenosis (ABSORB II): a 3 year, randomised, controlled, single-blind, multicentre clinical trial. Lancet. 2016; 388(10059): 2479-2491, doi: 10.1016/S0140-6736(16)32050-5, indexed in Pubmed: 27806897.
10. Wykrzykowska J, Kraak R, Hofma S, et al. Bioresorbable Scaffolds versus Metallic Stents in Routine PCI. N Engl J Med. 2017; 376(24): 2319-2328, doi: 10.1056/nejmoa1614954.

11. Serruys PW, Onuma Y, Dudek D, et al. Evaluation of the second generation of a bioresorbable everolimus-eluting vascular scaffold for the treatment of de novo coronary artery stenosis: 12-month clinical and imaging outcomes. J Am Coll Cardiol. 2011; 58(15): 1578-1588, doi: 10.1016/j.jacc.2011.05.050, indexed in Pubmed: 21958884.

12. Puricel S, Cuculi F, Weissner M, et al. Bioresorbable coronary scaffold thrombosis: multicenter comprehensive analysis of clinical presentation, mechanisms, and predictors. J Am Coll Cardiol. 2016; 67(8): 921-931, doi: 10.1016/j.jacc.2015.12.019, indexed in Pubmed: 26916481.

13. Gutiérrez-Chico JL, Cortés C, Schincariol M, et al. Implantation of bioresorbable scaffolds under guidance of optical coherence tomography: Feasibility and pilot clinical results of a systematic protocol. Cardiol J. 2018; 25(4): 443-458, doi: 10.5603/ CJ.a2018.0055, indexed in Pubmed: 29774520.

14. Räber L, Brugaletta S, Yamaji K, et al. Very late scaffold thrombosis: intracoronary imaging and histopathological and spectroscopic findings. J Am Coll Cardiol. 2015; 66(17): 1901-1914, doi: 10.1016/j.jacc.2015.08.853, indexed in Pubmed: 26493663.

15. Onuma Y, Serruys PW, Perkins LEL, et al. Intracoronary optical coherence tomography and histology at 1 month and 2, 3, and 4 years after implantation of everolimus-eluting bioresorbable vascular scaffolds in a porcine coronary artery model: an attempt to decipher the human optical coherence tomography images in the ABSORB trial. Circulation. 2010; 122(22): 2288-2300, doi: 10.1161/ CIRCULATIONAHA.109.921528, indexed in Pubmed: 20975003.

16. Aoki J, Nakazawa G, Tanabe K, et al. Incidence and clinical impact of coronary stent fracture after sirolimus-eluting stent implantation. Catheter Cardiovasc Interv. 2007; 69(3): 380-386, doi: 10.1002/ccd.20950, indexed in Pubmed: 17195199.

17. Lee MS, Jurewitz D, Aragon J, et al. Stent fracture associated with drug-eluting stents: clinical characteristics and implications. Catheter Cardiovasc Interv. 2007; 69(3): 387-394, doi: 10.1002/ccd.20942, indexed in Pubmed: 17195203.

18. Kuramitsu S, Hiromasa T, Enomoto S, et al. Incidence and clinical impact of stent fracture after everolimus-eluting stent implantation. Circ Cardiovasc Interv. 2012; 5(5): 663-671, doi: 10.1161/CIRCINTERVENTIONS.112.969238, indexed in Pubmed: 23011266.

19. Wu X, von Birgelen C, Muramatsu T, et al. A novel four-dimensional angiographic approach to assess dynamic superficial wall stress of coronary arteries in vivo: initial experience in evaluating vessel sites with subsequent plaque rupture. EuroIntervention. 2017; 13(9): e1099-e1103, doi: 10.4244/EIJ-D-16-01020, indexed in Pubmed: 28262624.

20. Wu X, von Birgelen C, Li Z, et al. Assessment of superficial coronary vessel wall deformation and stress: validation of in silico models and human coronary arteries in vivo. Int J Cardiovasc Imaging. 2018; 34(6): 849-861, doi: 10.1007/s10554-018-1311-7, indexed in Pubmed: 29397475.

21. Gutiérrez-Chico JL. Superficial wall stress: the long awaited comprehensive biomechanical parameter to objectify and quantify our intuition. Int J Cardiovasc Imaging. 2018; 34(6): 863-865, doi: 10.1007/s10554-018-1386-1, indexed in Pubmed: 29876693. 